Cahiers, Cahiers balkaniques

Balkaniques

Hors-série | 2016

Manger en Grèce

\title{
Jeûner en Grèce
}

Pratiques religieuses et traditions populaires

To do Lent in Greece: Religion and Popular Practices

H v

\section{Georges Kostakiotis}

\section{QpenEdition \\ Journals}

Édition électronique

URL : https://journals.openedition.org/ceb/7206

DOI : $10.4000 /$ ceb.7206

ISSN : 2261-4184

Éditeur

INALCO

Édition imprimée

ISBN : 978-2-85831-230-6

ISSN : 0290-7402

\section{Référence électronique}

Georges Kostakiotis, « Jeûner en Grèce », Cahiers balkaniques [En ligne], Hors-série | 2016, mis en ligne le 04 février 2016, consulté le 06 juillet 2021. URL : http://journals.openedition.org/ceb/7206 ; DOI : https://doi.org/10.4000/ceb.7206

Ce document a été généré automatiquement le 6 juillet 2021

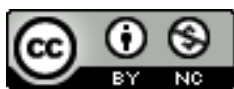

Cahiers balkaniques est mis à disposition selon les termes de la Licence Creative Commons Attribution - Pas d'Utilisation Commerciale 4.0 International. 


\title{
Jeûner en Grèce
}

\author{
Pratiques religieuses et traditions populaires \\ To do Lent in Greece: Religion and Popular Practices

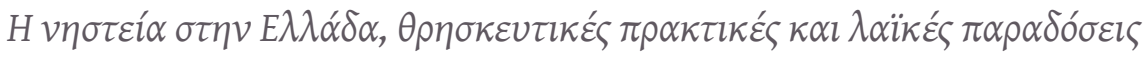

\section{Georges Kostakiotis}

1 À partir de textes néotestamentaires et d'écrits patristiques, nous tâcherons, dans cette communication, de percevoir la prescription de l'interdit alimentaire dans la société grecque telle qu'elle se révèle dans la tradition populaire et se cristallise dans la littérature pour caractériser une région, un espace, une culture.

2 Loin d'être associée au jeûne, l'alimentation occupe une place importante dans les textes du Nouveau Testament. Les repas organisés pour des célébrations festives ou pour se retrouver à l'occasion des commémorations font partie du quotidien du Christ et de ses disciples. Ce n'est pas par hasard si le premier miracle du Christ, celui qui va annoncer son enseignement public, se réalise à l'occasion du festin des Noces de Cana (JEAN, 2, 1-12); il est de plus associé à la boisson, c'est-à-dire au plaisir d'accompagner le repas. Ce n'est pas anodin non plus si la dernière réunion du Christ avec ses disciples est un banquet : la Cène (LUC, 22, 8-38). Notons également la multiplication des pains et des poissons qui tient une place importante parmi les miracles dans les écritures évangéliques (MATTHIEU, 14, 14-21 et 15, 32-38).

3 Il est clair que la nourriture, qu'elle soit considérée comme un moment de rassemblement festif, de célébration cérémoniale ou de simple besoin, n'est ni critiquée ni déconsidérée pour être mise à l'écart. Manger dans la tradition des Évangiles occupe une place d'exception dans les moments heureux et importants de la vie humaine et signifie non seulement se rassasier, mais aussi fêter, célébrer, se rassembler. Le Christ et ses proches y participent et y contribuent. Rappelons à ce propos que Platon au cours du Banquet invite les convives à disserter sur l'amour. Dieu-Amour se révèle dans l'acte même de manger ; manger est sacré, car ce qui maintient en vie est sacré (BAUD, 2014, 8) et Dieu maintient en vie!

4 Les références que nous avons sur la privation alimentaire concernent la vie de saint Jean Baptiste et la retraite de Jésus lui-même dans le désert. Dans ces deux cas, il 
est question d'un contrôle strict de l'alimentation qui est associée, de ce fait, à un mode de vie austère, d'ascèse accompagné de prières. À ce stade, il ne s'agit point d'une vie proposée comme exemplaire aux chrétiens ; les Évangélistes n'envisagent pas ces deux passages comme une série de restrictions à appliquer. Il s'agit plutôt de rites de passage - rites d'initiation fréquents dans certaines sociétés juives de l'époque du Christ (DANIÉLOU, 1995, 13-22).

5 C'est plus tard, avec les Épitres de saint Paul $(1$, Corinthiens, 8, 8) que le fait de s'abstenir de consommer certains aliments prendra la forme d'une interdiction pour les nouvelles communautés chrétiennes. Dans ces écrits, la privation joue un rôle éducatif et elle est toujours associée à d'autres mesures qui concernent l'ensemble de la communauté dans le but d'une part, de la faire se démarquer du reste de la société et d'autre part, de tisser des liens forts entre ses membres.

6 Nous pouvons désormais parler d'une série de rituels associés aussi à la nourriture qui se transforment en des phénomènes sociétaux d'identification permettant de repérer d'abord des groupes humains et ensuite des sociétés ${ }^{1}$. Ainsi le chrétien est appelé à respecter certaines pratiques qui, faisant référence à la retraite du Christ, prennent des dimensions sociales basées d'une part, sur le caractère et le tempérament dur de saint Paul et d'autre part, sur les besoins réels de ces gens qui, pour la plupart, vivaient en marge de la société et qui étaient souvent des pauvres. C'est justement dans ce contexte également qu'il faudrait comprendre le "c'est pourquoi, si un aliment scandalise mon frère, je ne mangerai jamais de viande, afin de ne pas scandaliser mon frère $»^{2}(1$, Corinthiens, 8,13$)$. Éviter les discriminations des moins favorisés tout en assurant la paix sociale dans le respect de la sensibilité de tous a été la principale préoccupation de la première église.

7 Par ailleurs, la nourriture reste toujours un moyen de se retrouver, mais cette fois l'accent est mis sur le partage à travers les agapes. Le riche de la parabole (LUc, 16, 19-31) ne va pas en enfer parce qu'il mange beaucoup, mais parce qu'il refuse de partager en laissant le pauvre Lazare affamé.

8 C'est avec les écrits des Pères de l'Église que ces «restrictions» de saint Paul se formalisent pour s'intégrer dans la vie des chrétiens en tant que règles à suivre. Au quatrième siècle, saint Jean Chrysostome et saint Basile soulignent à plusieurs reprises dans leurs écrits l'importance du jeûne, mais insistent sur le fait que s'abstenir de certains aliments ne doit pas être vu comme un but en soi et que le jeûne seul ne suffit pas pour déterminer la relation des chrétiens avec le Christ. Il ne s'agit que "d'une étape $»^{3}(\mathrm{PG}, 56,139)$ dans la vie de l'Église et fait partie d'un ensemble de savoir-être qui détermine la vie des gens quotidiennement ( $P G, 53,74,75)$. En tant que tel, saint Jean Chrysostome n'hésite pas à qualifier le jeûne de «mère de tous les biens et maitre de la sagesse et de toute autre vertu $»^{4}(\mathrm{PG}, 53,22)$. Pour saint Basile le jeûne est considéré comme un exercice qui permet à l'homme de maitriser sa volonté et ses besoins matériels pour devenir ensuite un outil qui lui permettra de gérer la pensée et le désir (PG, 31, 163-198).

9 Pour les Pères de l'Église, il ne s'agit pas d'une privation, mais d'un choix délibéré qui apaise l'esprit, renforce le caractère et permet à l'homme de garder le contrôle de soi et de lui faciliter les relations sociales : autrement dit, il devient la base pour constituer l'Église (église = relations). Les Pères suivent une argumentation qui leur permet de justifier l'importance du jeûne sans pour autant l'isoler de l'ensemble de la vie quotidienne. De cette façon, le jeûne sans être considéré comme un comportement à 
part trouve sa place dans l'ensemble de la vie humaine personnelle ou sociale, le koinon et le idion, de Lévi-Strauss $(2010,318)$.

Un peu plus tard, saint Jean Climaque continue sur cette même problématique; lui aussi considère le jeûne comme une étape dans la volonté du chrétien de maîtriser les passions et de se forger un caractère solide et pur (Échelle, 14, 17-19). Mais petit à petit chez lui, la nourriture acquiert une fonction endoscopique (Échelle, 14, 30) où l'intériorité n'est plus un sujet: l'alimentation désormais doit être vue comme une des tesselles qui contribuent à la constitution de l'expérience directe de l'entour de l'homme pour aborder le milieu objectif qu'il découvre (BARTHES, 1981, 42, 43). L'être est dans le monde si incertain qu'il a besoin de se projeter hors de soi (BATAILle, 2014, 98). Or, les aliments aussi se transforment en des objets qui le (l'être) font glisser du plan extérieur à l'intériorité du sujet (Bataille, 2014, 28) pour le rendre objet. Dans la profondeur de l'âme, l'homme s'engage dans un face à face avec Dieu (BARTHES, 1981, 42, 43). C'est ainsi que saint Jean arrive désormais à la vision de Dieu ${ }^{5}$.

11 C'est pour cette raison que sur les trente marches qui constituent son échelle des vertus, le combat contre la gourmandise ${ }^{6}$ se trouve en quatorzième position, occupant ainsi une place centrale et charnière dans la lutte de l'homme contre les vices durant son parcours vers Dieu. Notons à ce propos que saint Jean place l'étape du jeûne juste avant celle de la pureté pour l'associer ouvertement au combat contre la volupté, la luxure et les vices charnels.

12 Nous insistons sur cette perspective, car c'est ainsi qu'elle a été adoptée par les sociétés et conservée à travers la tradition populaire jusqu'à nos jours. Tel est le témoignage apporté par les chansons traditionnelles où la période du carême est clairement associée à l'abstinence sexuelle. D'une part, il est question de jeûne : "Bienvenue au Carême avec l'ail et la laitue $»^{8}$ (SAMIOU, 1998, 29) et d'autre part, d'absence de tout contact physique; durant les sept semaines qui précèdent Pâques les jeunes gens, non mariés, n'ont même pas le droit de s'adresser la parole : « Durant sept semaines ${ }^{9}$ mon petit oiseau, je ne t'ai pas adressé la parole pour ne pas t'entraîner dans le péché, car c'était le carême $»^{10}$ (SAMIOU, 1998, 81). La dureté de la vie et la rigidité de la mentalité des sociétés rurales semblent trouver leur expression à travers les pratiques religieuses, transformées désormais en coutumes.

La communauté, dans sa volonté de contrôler toute forme de liberté, toute menace de l'ordre établi, adopte une série de rituels qui en apparence se conforment aux convictions chrétiennes, mais qui, en fin de compte, se trouvent dénuées de toute croyance consciente. La soumission au code social est totale. Cela ne veut pas dire pour autant que l'individu ne tente pas d'y échapper: avec la rupture du jeûne la fête commence, les couples se forment à nouveau pour conclure cette fois-ci leurs relations : "Le jour des Pâques est beau et nous faisons la fête " ${ }^{11}$, la succession des subjonctifs du verbe être, précédé par l'impératif du verbe venir: «Viens, que tu sois, que je sois, que tu sois (= pour être ensemble) $»^{12}$ serait une allusion à l'acte sexuel, tandis que les subjonctifs continus qui suivent ne laissent point de doute: «que je te prépare le lit pour dormir $»^{13}$ (SAMIOU, 1998, 81).

14 Le monde se construit comme un huis clos où la dureté (pour ne pas parler de violence psychologique) d'une société verrouillée de l'intérieur devient signe identitaire. Nous voulons dire que le caractère préparatif et purifiant du carême proposé par l'Église se transforme en toutes sortes d'interdictions qui déterminent désormais la vie des sociétés fermées dans la volonté de se préserver et se distinguer des autres. Le jeûne 
aussi est invité à jouer ce rôle puisqu'il constitue, au-delà de la création des liens entre les membres du même groupe, un moyen pour se différencier aussi de l'autre, de l'inconnu, de l'étranger et donc de l'ennemi. Lévi-Strauss parle de l'hétérogénéité $(2010,318,319)$. Ainsi, la question de l'identité se réalise aussi avec l'alimentation pour s'inscrire dans un ethnocentrisme dont les limites deviennent à la fois infranchissables et difficiles à distinguer ${ }^{14}$.

Cette utilisation du jeûne, transformé en signe identitaire, est exploitée par Stratis Doukas dans l'Histoire d'un prisonnier. L'écrivain nous place du côté opposé : celui qui doit jeûner n'est pas le chrétien, mais le musulman ; avec le Moi qui devient Autre, le protagoniste devient l'exclu d'une société qui trouve son expression identitaire à travers la pratique du jeûne; et, bien que le héros ait la possibilité de ne pas jeûner puisqu'il est loin de chez lui, dans la volonté de prouver son appartenance à la communauté turque il propose lui-même de jeûner (DouKAS, 2008, 45, 46). Le problème n'est pas l'introspection des motifs du narrateur ni les effets que la narration produit sur le lecteur; il est de décrire le code à travers lequel les personnages sont signifiés à travers l'épisode du jeûne. Il ne s'agit donc pas uniquement d'enrichir la notion d'identité, mais aussi de déconstruire ce concept en ces multiples occurrences et de briser certaines relations de surface bien établies (LÉVI-STRAUSS, 2010, 319). Les signes envoyés par le narrateur paraissent désormais renversés face à ceux du Moi transformé (BARTHES, 1977, 38). Stratis Doukas exploite les limites de l'identité nationale ou ethnique qui, une fois de plus, se confond avec celle de la religion pour arriver à se déterminer. La pratique religieuse dénuée de toute croyance devient un mécanisme pour affirmer son appartenance - une pratique qui redevient d'actualité dans les sociétés d'occident, dites plurielles - ethnique ou nationale. Lorsqu'il ne suffit pas de dire son appartenance, mais il faut le prouver, c'est le jeûne qui vient confirmer l'identité. Le Turc de Stratis Doukas respectera le jeûne de cette année, car les infidèles sont partis; de son côté, le Grec fera de même pour prouver qu'il est réellement Turc.

On pourra remarquer que lorsque la nourriture, les plats cuisinés ne permettent plus l'identification d'une communauté, puisque cette nourriture appartient aussi à d'autres, c'est l'absence de nourriture, le jeûne, qui va permettre l'identification ou le sentiment d'appartenance à une société. Phénomène sociétal le jeûne? Probablement puisqu'il faudra distinguer le jeûne du carême, celui du ramadan, et bien sûr ceux liés aux diverses modes diététiques d'aujourd'hui.

Cette absence de croyance réelle et consciente en la pratique religieuse se banalise, les héros se décomplexent et le jeûne devient enfin une source de critique ou de dérision. Pour Maria Iordanidou, dans son roman Loxandra où la cuisine tiendra une place centrale, le jeûne sera vu comme un synonyme de l'absence de goût que la protagoniste doit rompre pour vérifier l'assaisonnement de sa préparation (IORDANIDOU, 1999, 24); de même pour le carême qui permettra à l'auteur d'ironiser sur la cuisine et le goût anglais :

Alors, nous sommes allés dîner, je vois qu'on nous apporte un beau plat en argent sous cloche. Je me suis dit: ah! la! la! qu'est-ce que cela peut bien être; ils soulèvent le couvercle et que vois-je ? Des pommes de terre à l'eau! Georges, je dis à ce balourd, est-ce que les Anglais font le carême? Tu ne leur demanderais pas un peu d'huile et de vinaigre pour que je trempe mon bout de pain $?^{15}$ (IORDANIDOU, 1999, 161, 162) toujours le drame des héros de Maria Iordanidou qui cherchent obstinément à 
construire leur identité par la négation des autres. Les personnages enfermés encore dans une sorte d'impasse historique tentent désespérément de répondre à la question qui suis-je, incapables d'envisager l'hétérogénéité de Lévi-Strauss comme une réalité possible. La mise en question de la subjectivité, de la personne et du groupe semble impossible. Pour avoir des tentatives de réflexion sur la question dialectique que faire et non pas qui suis-je, il faudra attendre encore (BARTHES, 1981, 110, 111 ; LÉVI-STRAUSS, 2010, $318,319)$.

19 Les protagonistes pratiquent le jeûne par intérêt ou par habitude sans convictions profondes, comme signes extérieurs d'une appartenance culturelle -identitaire. Pourtant ils exigent que leur clergé reste bien attaché à ces pratiques et respectueux de ces traditions; et si ce n'est pas ainsi, ils sont scandalisés. C'est le cas des héros de Giorgos Skambardonis dans sa nouvelle Boisson Chaude ${ }^{16}(2003,67-69)$ qui sont choqués lorsqu'ils voient que le moine, un jour de jeûne, prépare du lait. Ou bien du jeune moine apprenti dans Consommation de poisson ${ }^{17}(1997,13-18)$ qui risque de s'étouffer lorsqu'il mange du poisson. L'écrivain, dans la perspective nouvelle d'une quête de spiritualité, retrouve l'authenticité de certaines pratiques religieuses comme celle du jeûne à travers ces religieux athonites qui semblent vivre l'abstention alimentaire comme un besoin intérieur qui complète leur pensée et leur mode de vie.

Si les autorités, les valeurs traditionnelles, n'ont plus depuis longtemps de sens pour un grand nombre, la critique à laquelle la tradition a succombé ne peut être indifférente à ceux dont l'intérêt est l'extrême du possible (BATAILLE, 2014, 20). Ce sera le cas du père du monastère de Karakallou dans Père Luc, le très humble ${ }^{18}(1992,89,90)$ qui se contente de pain et de salade, dort par terre refusant le confort du lit et se couvre de sa seule soutane. Pour Skambardonis l'expérience intérieure devient à nouveau l'autorité (la force) qui rend possible l'accomplissement d'être face à Dieu : «soi-même, ce n'est pas le sujet s'isolant du monde, mais un lieu de communication, de fusion du sujet et de l'objet » (BATAILLE, 2014, 21).

21 Avec ces différents exemples choisis, nous pouvons observer une évolution progressive du champ sémantique du jeûne dans le monde chrétien de l'espace grec. Le jeûne prend la fonction d'un signe pour se transformer d'abord en symbole qui va déterminer le périmètre de la société d'appartenance et ensuite, acquérant une sorte de mémoire distinctive, va s'opposer aux autres sociétés qui n'ont pas la même mémoire et donc forment un autre système (BARTHES, 1981, 214-216). La mise en place des premières règles par saint Paul et l'institution établie par les Pères au $\mathrm{IV}^{\mathrm{e}}$ siècle se transforment peu à peu en fondements de la construction de l'identité culturelle et nationale jusqu'à devenir des Formes sans fond ${ }^{19}$ dans les sociétés plus récentes.

La conscience symbolique voit le signe dans sa dimension profonde, c'est dire que le jeûne prend un sens vital en tant que choix volontaire et libre, car il s'agit, dans la conception de l'Église accomplie, moins d'une forme de communication que d'un instrument de participation. Mais lorsque la société prend conscience de son " existence en tant que telle" face à une autre, le signifié ne conserve qu'un rôle démonstratif qui a tendance à se vider de sa consistance sans pour autant perdre sa signification (BARTHES, 1981, 217) pour enfin devenir le nouveau départ face à un certain besoin de revenir aux sources. 


\section{BIBLIOGRAPHIE}

BARTHES Roland, 1981, Essais critiques, Paris : Seuil-Points-Essais.

BARTHES Roland, 1977, « Analyse structurale des récits » in Poétique du récit, Paris : Seuil-Points-

Essais, p. 9-57.

BATAILLE Georges, 2014, l'Expérience intérieure, Paris : Tel-Gallimard.

BAUD Philippe, 2014, Et Dieu dit : Passons à table! Paris-Montréal : Médiaspaul.

DANIÉLOU Jean, 1995, les Manuscrits de la mer Morte, Paris : Éditions de l’Orante/Seuil.

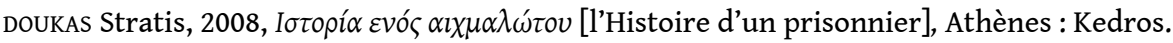

IORDANIDOU Maria, 1999, $\Lambda \omega \xi \alpha ́ v \tau \tau \rho \alpha$ [Loxandra,], Athènes : Estia.

LÉVI-STRAuss Claude (dir.), 1983, l’Identité, Paris : Puf-Quadrige.

MAIORESCU Titu, 1978, În contra direcȚiei de astăzi în cultura română [Contre l'orientation actuelle de la culture roumaine] in Euvres complètes, vol. I, Bucarest : Univers Enciclopedic.

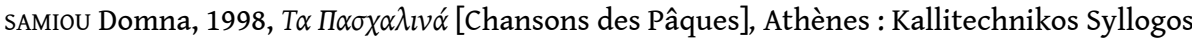

Dimotikis Mousikis Domna Samiou.

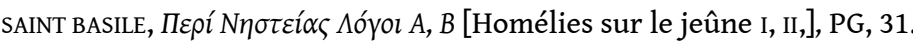

SAINT JEAN CHRYSOSTOME, Euvres Complètes, PG 53 et 56.

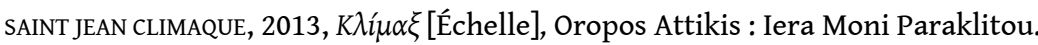

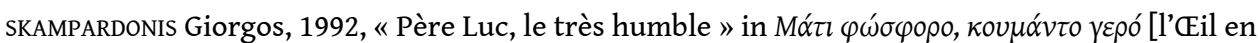
phosphore, commandement solide], Athènes : Kastaniotis.

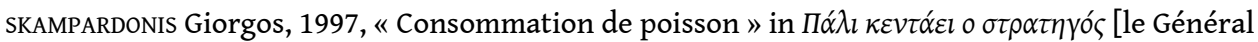
brode à nouveau], Athènes : Kastaniotis.

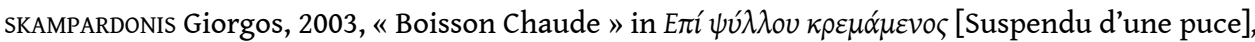
Athènes : Kastaniotis.

\section{NOTES}

1. Lévi-Strauss dans Tristes Tropiques évoque le rituel du maté en Amérique du Sud, de la feuille de coca mâchée dans les Andes, du kat au Yémen, des prohibitions alimentaires qui prennent une signification sociologique pour caractériser des positions hiérarchiques dans une communauté. Il s'agit d'observations qui conduisent à repérer des groupes et des sociétés.

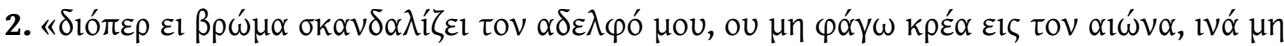

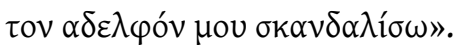

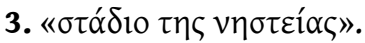

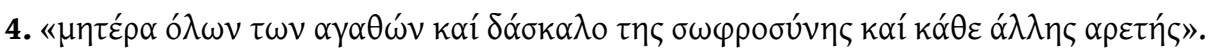

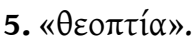




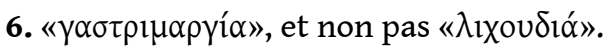

7. « $\alpha \gamma \nu \varepsilon i ́ \alpha »$.

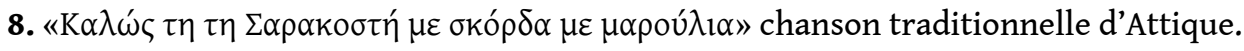

9. Le Carême chez les orthodoxes dure 50 jours (sept semaines) et non pas 40.

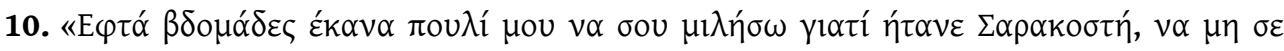

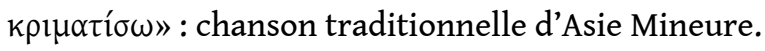

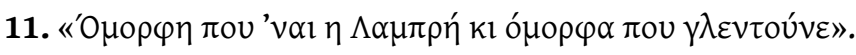

12. «'E $\lambda \alpha v \alpha$ ' $\sigma \alpha l, v \alpha$ ' $\mu \alpha l, v \alpha$ ' $\sigma \alpha l »$.

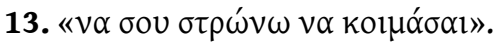

14. Lévi-Strauss note que « un moyen de dépasser leurs limites, soit en s'étendant à des domaines extérieurs à l'ensemble initial, par universalisation, soit par particularisation, en prolongeant la démarche classificatrice au-delà de ses bornes naturelles, c'est-à-dire jusqu'à l'individualisation ", (2010, 319).

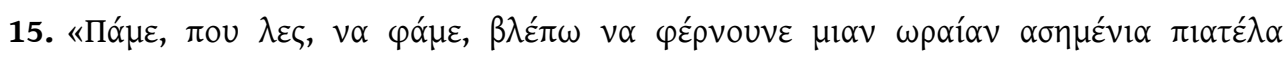

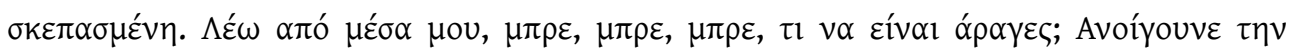

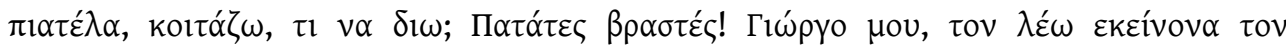

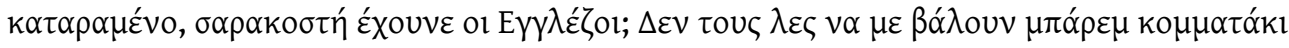

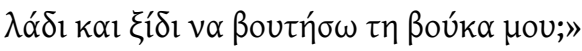

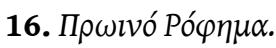

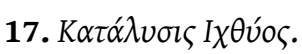

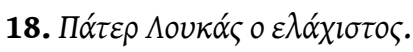

19. Formele fără fond selon la théorie de Titu Maiorescu $(1978,153)$.

\section{RÉSUMÉS}

Si la nourriture détermine les relations humaines et se transforme en code pour ensuite les représenter, dans quelle mesure les carêmes et le jeûne en général, contribuent-ils à la formation d'une identité sociale, sinon nationale ? Puisque toute pratique gastronomique se construit à la fois dans le discours social et dans le cadre sociétal, quelle est la place de l'alimentation à travers les pratiques religieuses dans les sociétés orthodoxes grecques?

Dans notre communication, nous tâcherons d'apercevoir la réception de l'interdit alimentaire dans la société grecque telle qu'elle se reflète dans la littérature et se cristallise dans la tradition populaire pour caractériser une région, un espace, une culture. Nous étudierons comment dans le monde grec, différents types de nourriture réactualisent constamment la complexité des formes de stratification sociale à travers les enjeux culturels et fixent les rapports sociaux ainsi que les jeux de pouvoir.

If nutrition determines human relationships and transforms into a code to then represent them, to what extent do Lent and fasting in general, contribute to the formation of a social or even a national identity? Since any gastronomic practice is built both by social discourse and the societal framework what is the place of food across religious practices in the Greek Orthodox 
societies?

Having as a starting point the new-Testament texts and patristic writings, in our communication we will try to see the reception of the food ban in the Greek society as it is reflected in the literature and is solidified in the popular tradition to characterize a region, a space, a culture. We will study how in the Greek world, different types of food constantly re-actualize the complex forms of social stratification through cultural issues and establish social relations and power games.

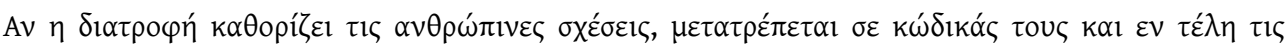

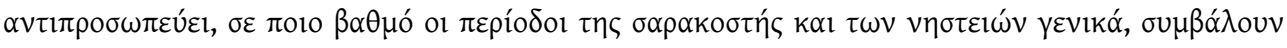

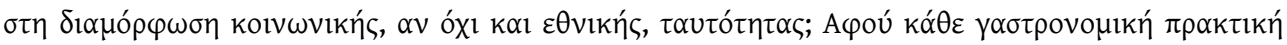

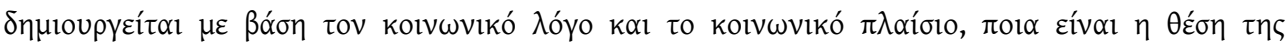

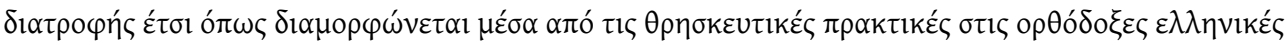

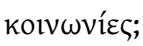

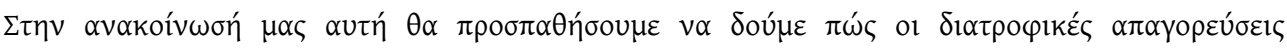

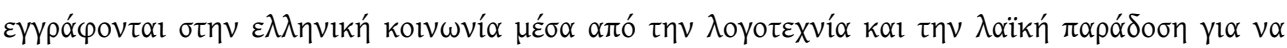

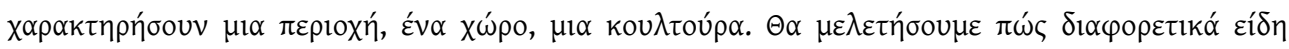

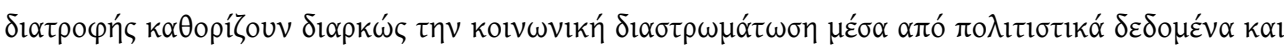

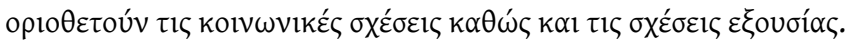

\section{INDEX}

Index géographique : Grèce

Thèmes : Société, Littérature

Mots-clés : carême, jeûne, nourriture, identité, tradition

Keywords : Lent, Fasting, Food, Identity, Greece, Society, Literature, Tradition

motsclesmk ПОЗАЈМИ, ПОСТОТ, ХРАНА, ИДЕНТИТЕТ, ТРАДИЦИЈА, ГРЦИЈА, СОЦИЈАЛНАТА, ЛИТЕРАТУРА

motsclestr Büyük Perhiz, Oruç tutma, Yiyecek, Kimlik, Genelek, Yunanistan, Toplum, Edebiyat

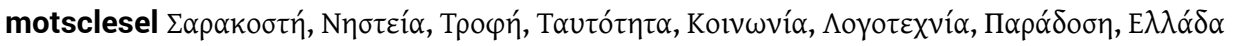

\section{AUTEUR}

\section{GEORGES KOSTAKIOTIS}

CREE, Inalco, Sorbonne Paris Cité, 2 rue de Lille 75343 PARIS cedex 07. 